Markus Dreßler*

\title{
Religion und religiöse Tradition: Unterscheidungsdiskurse zu den Grenzen des Islams
}

https://doi.org/10.1515/zfr-2018-0023

Zusammenfassung: Dieser Beitrag beschäftigt sich am Beispiel des islamischen Diskursfeldes mit der metasprachlichen Beschreibung objektsprachlicher Abund Ausgrenzungsdiskurse. Dies bringt zwangsläufig eine Auseinandersetzung mit den Dynamiken zwischen objekt- und metasprachlicher Begriffsebene mit sich. Der Artikel diskutiert in einem ersten Schritt Talal Asads Konzeption islamischer Orthodoxie und den ihr zugrundeliegenden Traditionsbegriff. In einem zweiten Schritt werden anhand von Beispielen aus dem nordamerikanischen Sufidiskurs und dem modernen türkischen Religionsdiskurs objektsprachliche Grenzziehungsdynamiken innerhalb des islamischen Diskursfeldes analysiert. Der letzte Teil des Aufsatzes widmet sich der Metaebene und führt, in Abgrenzung zum statischen Stammbaummodell, einen dynamischen Begriff von religiöser Tradition ein, der es ermöglichen soll, Überlagerungen zwischen religiösen Formationen niedrigen Verdichtungsgrades sowie Prozesse der Religionisierung ins Blickfeld zu nehmen.

Schlagwörter: Religionsdiskurse, Objekt- und Metasprache, religiöse Tradition, innerislamische Differenzierung, Talal Asad, Religionisierung, Islam, Sufismus, Aleviten

Abstract: Drawing on the example of the discursive field of Islam, this article poses the question of how scholarly discourse can represent object language discourses of religious distinction. This necessarily requires engagement with the intricate dynamics between object language and metalanguage. As a first step, the article discusses Talal Asad's conception of Islamic orthodoxy and its underlying concept of discursive tradition. Drawing on examples from North American Sufi discourses and the modern Turkish discourse on religion, the text discusses, as a second step, the dynamics of object language boundary construction and the confinement of "Islam" that it produces. The final part of the article introduces, in

*Kontaktperson: PD Dr. Markus Dreßler, Universität Leipzig, KFG „Multiple Secularities Beyond the West, Beyond Modernities“, Universität Leipzig, Nikolaistraße 8-10, 04109 Leipzig, E-Mail: markus.dressler@uni-leipzig.de 
contradistinction to the static family tree model, a dynamic concept of religious tradition, which enables us to focus on the overlaps and interactions between religious formations of low density and on processes of religionization.

Keywords: religion discourse, object language and metalanguage, religious tradition, inner-Islamic difference, Talal Asad, religionization, Islam, Sufism, Alevism

Beschäftigt man sich mit Gruppierungen am Rande des islamischen Diskursfeldes, dann wird man immer wieder mit der Frage der Islamizität bestimmter umstrittener Praktiken und Vorstellungen oder ganzer Gemeinschaften konfrontiert. Die Auseinandersetzung mit den Grenzen „des Islams“ und der Frage der Islamizität verschiedener Interpretationen innerhalb des islamischen Diskursfeldes begleitet einen dabei sowohl auf der Ebene der Objektsprache als auch auf der Ebene der Metasprache. In diesem Beitrag geht es zunächst darum, wie man objektsprachliche Ab- und Ausgrenzungsdiskurse metasprachlich sinnvoll abbilden kann. Dies bringt zwangsläufig eine Auseinandersetzung mit den Dynamiken zwischen objekt- und metasprachlicher Begriffsebene mit sich. Vorliegender Artikel diskutiert in einem ersten Schritt Talal Asads Konzeption islamischer Orthodoxie und den ihr zugrundeliegenden Traditionsbegriff. Das Hauptaugenmerk liegt hierbei darauf, den Islam metasprachlich als historische Realität zu fassen, ohne dabei selbst normierend in den Diskurs über den Islam einzugreifen. In einem zweiten Schritt werden, anhand von Beispielen aus dem nordamerikanischen Sufidiskurs und dem modernen türkischen Religionsdiskurs, objektsprachliche Grenzziehungsdynamiken innerhalb des islamischen Diskursfeldes diskutiert. Der dritte und letzte Teil des Aufsatzes wendet sich der Metaebene zu und führt, in Abgrenzung zum Stammbaummodell, einen dynamischen Begriff von religiöser Tradition ein. Dieser soll das Augenmerk einerseits auf Überlagerungen und Grenzbereiche zwischen religiösen Traditionen und andererseits auf Grade ihrer sozialen und diskursiven Verdichtung legen, wobei fortgeschrittene Prozesse solcher Verdichtung hier als Religionisierung verstanden werden. Aushandlungsprozesse in Bezug auf die Authentizität beziehungsweise Zugehörigkeit zu einer bestimmten religiösen Tradition werden dabei, angelehnt an Bourdieu und Boyarin, als wichtige Faktoren solcher Verdichtungsprozesse aufgefasst. 


\section{Islam als diskursive Tradition}

Ein einflussreicher Text, der in der wissenschaftlichen Diskussion um den Begriff des Islams als diskursiver Tradition bis heute teilweise kontrovers diskutiert wird, ist Talal Asads The Idea of an Anthropology of Islam (im Folgenden: AoI). Der Text ist für unseren Zusammenhang von besonderem Interesse, da er sich direkt und kritisch mit der metasprachlichen Beschreibung des Islams auseinandersetzt:

„If one wants to write an anthropology of Islam one should begin, as Muslims do, from the concept of a discursive tradition that includes and relates itself to the founding texts of the Qur'an and the Hadith. Islam is neither a distinctive social structure nor a heterogeneous collection of beliefs, artifacts, customs, and morals. It is a tradition.“ (Asad 1986, 14)

Asads Intervention kann als Anstoß zu einer anthropologischen Wende in der wissenschaftlichen Auseinandersetzung mit dem Islam verstanden werden: Orientiert an den autoritativen Referenztexten der sunnitischen Tradition, Koran und Hadith, und in Distanz zu extrinsischen Konzepten herkömmlicher westlicher Islamforschung. Mit seinem Fokus auf (Religions-)Praxis kann der Text gleichzeitig auch als Reaktion auf den linguistic turn in den Kultur- und Sozialwissenschaften verstanden werden (Iqbal 2017, 195). Seinen begrifflichen Ausdruck findet dies im Konzept der diskursiven Tradition, die sich immer auf Formen von Praxis bezieht:

„A tradition consists essentially of discourses that seek to instruct practitioners regarding the correct form and purpose of a given practice that, precisely because it is established, has a history. [...] An Islamic discursive tradition is simply a tradition of Muslim discourse that addresses itself to conceptions of the Islamic past and future, with reference to a particular Islamic practice in the present.“ $($ Asad 1986, 14)

Was unter Bezugnahme auf unterschiedliche autoritative Institutionen in diskursiven Aushandlungsprozessen als islamisch tradiert wird, konstituiert in Asads Lesart die islamische Orthodoxie: „Wherever Muslims have the power to regulate, uphold, require, or adjust correct practices, and to condemn, exclude, undermine, or replace incorrect ones, there is the domain of orthodoxy" (Asad 1986, 15). Orthodoxie, relational und nicht substanziell verstanden, wird von Asad des Weiteren beschrieben als Produkt eines allen diskursiven Traditionen gemeinen Bestrebens nach Kohärenz (Asad 1986, 16 f.).

1 Während Asad hier den Fokus auf Texte als Bezugspunkte für die Autorisierung islamischer Tradition legt, wandte er sich in seinen späteren Arbeiten, speziell in Genealogies of Religion, körperlichen Disziplinen als dem Ort zu, an dem Tradition autorisiert wird (Asad 1993; vgl. Scott und Hirschkind 2006, 8). 
Asad erkennt diskursive Verhandlungen von Fragen der Orthodoxie als Praxis an, über die Tradition gebildet wird. Gleichzeitig jedoch scheint sein Vorschlag, Hadith und Koran als die zentralen Bezugspunkte anzusehen, im Verhältnis zu denen islamische Tradition verhandelt wird, schon das Ergebnis dieses Aushandlungsprozesses (nämlich die hegemoniale sunnitische Orthodoxie) vorwegzunehmen (Wilson 2007, 184; Anjum 2007; Schrode 2010, 21-23). Die religiöse Normierung, die der Begriff ,islamische Tradition“ durch diese Auswahl mittelbar erfährt, wird in AoI nicht weiter thematisiert. In einer rezenten Diskussion ging Asad nochmals speziell auf diesen Punkt ein:

\begin{abstract}
„Disagreement is central to what I take a discursive tradition to be. This doesn't imply that all Muslims consciously try to follow the Qur'an and the Prophet's sayings, still less that all Muslims are familiar with them. It is that arguments about what it means to be a Muslim (when they do occur, and whether, when they occur, they invoke ,authorities' or not) are oriented towards a coherent understanding or appreciation of a divine revelation and of the role of the messenger who made it available to mankind.“ (Asad in Iqbal 2017, 200)
\end{abstract}

In Anbetracht dessen, dass es durchaus auch andere Institutionen gäbe, die man als Referenzpunkte islamischer Orthodoxie anführen könnte, bleibt der ausschließliche Bezug auf Koran und Hadith als Bezugspunkte islamischer Traditionsbildung meines Erachtens erklärungsbedürftig. Es finden sich in Asads Werk auch andere Stellen, in denen er sich dem Islam über einen impliziten Idealtyp des „frommen Muslims“ nähert, der wiederum über Bezug auf die kanonischen Autoritäten des sunnitischen Mainstreams gefasst wird. So führt Asad an anderem Ort, im Kontext einer kritischen Diskussion der Verwendung des Begriffs der Transzendenz für den islamischen Kontext, aus:

\begin{abstract}
„[A] pious Muslim would not use the word ,transcending but probably would echo the Qur'an and say, ,I have faith in God almighty and in the hereafter (al-äkhira). 'However, the meaning of what may be translated as, another world transcending this' for a pious Muslim is to be found partly in what he says about the Qur'an, in his invocations of it when speaking to other Muslims, and in his behavior toward the book as ,a sign from God' to his creatures in this world.“ (Asad 2001, 213 f.) $)^{2}$
\end{abstract}

Die Annahme eines Typus des „frommen Muslim“, der sich selbstredend über den Bezug zu Hadith und Koran innerhalb des islamischen Diskursfeldes positioniert, mag für viele empirische Kontexte heuristisch sinnvoll sein. Jedoch verengt er den Blick auf das islamische Diskursfeld und lässt insbesondere die immense Bedeu-

2 Für ein weiteres Beispiel normierender Rede über den Islam im selben Text siehe Asad 2001, 216 Anm. 29. 
tung nichtschriftlicher Autoritätsquellen innerhalb des islamischen Traditionsfeldes, zum Beispiel solche, die auf Abstammung und Charisma basieren, außer Acht (vgl. Dressler 2013a, 234-236).

Es besteht meines Erachtens eine gewisse Spannung zwischen der Bestimmung von Orthodoxie über autoritative Bezugspunkte islamischer Dogmatik und der genealogischen Methode, der es darum geht, diskursive Machtverhältnisse kritisch zu hinterfragen. Diese löst sich meines Erachtens auch unter Berücksichtigung der Verschränkung von Genealogie und traditionsfokussierter Methode, wie von Asad später beschrieben, nicht völlig auf (Asad 2006; Iqbal 2017). Auch David Scott (2006) verweist auf die inhärente Spannung zwischen Genealogie (inspiriert von Nietzsche und Foucault) und Tradition (inspiriert von Alasdair MacIntyre) in Asads Werk. Während Asad sich auf der einen Seite den genealogischen Skeptizismus zu eigen macht, im Gegensatz zur konventionellen Geschichtsschreibung, die Konstruktion von Kontinuitäten als Ausfluss hegemonialer Wissensregime hinterfragt und stattdessen den kontingenten Charakter der Gegenwart und seiner subjektbildenden Machtstrukturen hervorhebt, ${ }^{3}$ geht er doch nicht so weit, Geschichte an sich als analytisches Objekt in Frage zu stellen: Asad „,is also prompted by a counter-preoccupation with the ways in which historical forms of life, binding experience to authority, are built up over periods of time into regularities of practice, mentality, and disposition, and into specific conceptions of the virtues, and distinctive complexes of values" (Scott 2006, 140). Asad selbst erklärt seine Bezugnahme auf sowohl Genealogie als auch Tradition wie folgt:

„The latter [tradition, MD] sustains, elaborates, and sometimes argues over disciplinary practices; the former [genealogy, $\mathrm{MD}$ ] inquires into the contingent formation of their conditions of truthfulness. I do not use genealogy to uncover a succession of masks or to challenge the very idea of academic history. Nor do I regard tradition as mere continuity, mere imitation. [...] So, as I use it, genealogy is a way of (re)telling history by tracing contingencies that have come together to form an apparently natural development. [...] Tradition is primarily about practice, about learning the point of a practice and performing it properly and making it part of oneself, something that embraces Mauss's concept of habitus.“ (Asad 2006, 234)

Dennoch bleiben ein paar Fragezeichen. Bedient man sich eines genealogisch ausgerichteten Zugangs zum Begriff der Tradition, dann sollte sich die Bestimmung der Autoritäten, über die eine bestimmte Tradition als solche markiert wird, nicht auf diejenigen Instanzen beschränken, die eine hegemoniale Stellung inner-

3 Zur genealogischen Methode siehe Saar 2008; zu Religion und Genealogie einführend Schulze 2015, 109-116. 
halb einer Tradition erlangt haben. Da es der Genealogie essenziell um eine Subversion etablierter linearer Geschichtserzählungen und die Entlarvung des Wirkens von Machtstrukturen auf die Bildung von historischen Subjekten geht, müsste meines Erachtens verstärkt nach den Kontingenzen gefragt werden, durch die bestimmte Strukturen und Semantiken innerhalb einer Tradition eine hegemoniale Position erhalten konnten. Versteht man religiöse Traditionen im Kontext des bourdieuschen Feldbegriffs, dann erkennt man die konstitutive Bedeutung von Häresie, verstanden als subversive Infragestellung eines dominanten Religionsdiskurses, für die Formulierung und die Aufrechterhaltung bestimmter dogmatischer Positionen.

Bourdieu (1991) thematisiert das dialektische Verhältnis zwischen dominierenden und dominierten Positionen bei der Konstituierung und Aufrechterhaltung eines religiösen Feldes und der Bestimmung seiner Grenzen. Sein Zugang ist deshalb sehr gut für die Analyse der Grenzen von religiösen Traditionen geeignet. Zwar hat auch Asads diskursive Bestimmung von Orthodoxie ein konstruktivistisches Element, dieses wird jedoch durch die in AoI zunächst nicht weiter begründete Positionierung von Koran und Hadith als zentrale Bezugspunkte islamischer Traditionsbildung sowie anderenorts auch durch die Normierung eines frommen muslimischen Subjekts im Verhältnis zu islamischen Traditionsbildungsprozessen zumindest teilweise aufgehoben. Damit wird das islamische Diskursfeld gleichzeitig auch als religiöses Diskursfeld vordefiniert. Das ist bemerkenswert, da Asads genealogischer Zugang an sich stark an den kontingenten diskursiven Dynamiken interessiert ist, im Kontext derer Begriffe von Religion reifiziert werden. ${ }^{4}$

Der Ethnologe Samuli Schielke geht in seiner Kritik an Asad noch einen Schritt weiter. Er moniert, dass es bei der Formulierung der diskursiven Tradition, wie von Asad und anderen ihm folgend vorgenommen, nicht um eine diskursive Analyse des muslimischen Religionsfeldes gehe, sondern um einen Versuch, den Islam als Ganzes analytisch zu fassen (Schielke 2010, 4). In der Tat stellt Asad in AoI zu Beginn seiner Kritik diverser anthropologischer Zugänge zum Islam klar: „The argument here is not against the attempt to generalize about Islam, but against the manner in which that generalization is undertaken“ (Asad 1986, 5). Schielke argumentiert, dass die Formulierung einer islamischen diskursiven Tradition einen „focus on Islam as a peculiar entity“ impliziere und mithin trotz gegenteiliger Absicht de facto zu einer Verdinglichung des Islams beitrage. In der Folge würden Musliminnen und Muslime in einem Maße auf ihre Islamizität redu-

$4 \mathrm{Zu}$ Asads paradigmatischer Kritik am modernen Religionsbegriff siehe seine späteren Arbeiten, insbesondere Asad 1993 und 2003. 
ziert, das aus empirischer Sicht hinterfragt werden müsse (Schielke 2010, 5; siehe auch Houston 2009, 202). Das ist ein wichtiger Einwand. Auch Asad selbst betont: „Clearly, not everything Muslims say and do belongs to an Islamic discursive tradition“ (Asad 1986, 14).

Schielke artikuliert seinen Vorwurf, dass der Begriff einer diskursiven islamischen Tradition wie von Asad formuliert zu einer Verdinglichung des Islams beitrage, aus einer radikalkonstruktivistischen Position. Aus einer sozialkonstruktivistischen Perspektive würde ich hier jedoch entgegnen, dass es den Islam, beziehungsweise die islamische Tradition als sozial konstruierte Wirklichkeit, zweifelsohne gibt - und zwar unabhängig von dem realistischen Problem, ob man über den Islam „an sich“ etwas sagen kann. ${ }^{5}$ Diese sozial konstruierte Wirklichkeit des Islams unterliegt, da der Prozess sozialer Konstruktion ein dynamischer ist, einem ständigen Veränderungsprozess. Asads Verknüpfung von genealogischer Methodologie mit einem bestimmten Begriff von islamischer Tradition, der sich an der diskursiv etablierten Orthodoxie, wie sie heute weite Teile der islamischen Wirklichkeit dominiert, orientiert, ist meines Erachtens durchaus mit einem so verstandenen Sozialkonstruktivismus kompatibel. An anderer Stelle führt Asad aus:

„[T]he question arises among adherents as to which elements in the religious tradition are to be regarded as vital and which must be modified in order to maintain its continuity. The essence of each religion is thus not something unchanging and unchangeable but something that is at once to be preserved and defended as well as argued over and reformed in the changing historical circumstances that the tradition inhabits.” (Asad 2001, 208)

Asads Konzept der islamischen Tradition als diskursiver Aushandlungsprozess markierte eine wichtige Intervention in die Islamforschung. Die Frage ist gleichwohl, ob es hilfreich ist, sich bei der Konzeptualisierung der islamischen Tradition so stark an der etablierten Orthodoxie zu orientieren und die hegemoniale Bedeutung von Koran und Hadith als traditionsbildende Autoritäten zu übernehmen. Die in den nächsten Abschnitten dieses Aufsatzes diskutierten Beispiele (zu den Diskursen über den nordamerikanischen Sufismus beziehungsweise über die türkischen Aleviten) zumindest können mit einem so ausgerichteten Traditionsbegriff nicht adäquat gefasst werden.

Im Gegensatz zu einem in Bezug auf seine Referenzpunkte statischen Modell religiöser Tradition möchte ich deshalb in diesem Aufsatz einen Begriff von religiöser Tradition stark machen, der auf explizite Referenzpunkte zur Erklärung der Kontinuität einer Tradition verzichtet. Ein solcher Begriff religiöser Tradition, wie

5 Zur sozialkonstruktivistischen Epistemologie in der Religionsforschung siehe Dressler 2018. 
im dritten Teil des Aufsatzes ausgeführt wird, sollte nicht schon einen bestimmten Grad normativer Verfestigung voraussetzen, sondern die Herausbildung normierender Prozesse selbst in den Fokus nehmen. Es geht mir mithin um einen heuristischen Begriff religiöser Tradition, der es ermöglicht, verschiedene Verdichtungsgrade religiöser Traditionen zu unterscheiden ${ }^{6}$ und damit zur Analyse der Dynamik der Institutionalisierung von Religionen als sozialen Systemen beizutragen. Traditionen werden dabei als „Rede- und Handlungsgemeinschaft“ (Schulze 2015, 175) mit unterschiedlichen Graden der Institutionalisierung (bzw. Verdichtung) verstanden.

\section{Objektsprachliche Differenzierungsprozesse innerhalb von religiösen Traditionen}

\subsection{Muslim und non-Muslim Sufis, Sufi und non-Sufi Muslims}

Mein erstes Beispiel entnehme ich der innerislamischen Debatte über den Sufismus. Während meiner Forschungen zu nordamerikanischen Sufis (2002-2004) im Kontext des Forschungsclusters „Muslims in New York City“7 war ich von Beginn an mit dem Problem konfrontiert, Muslim Sufis klar von non-Muslim Sufis zu unterscheiden. Die Schwierigkeit ergab sich daraus, dass es in der Rezeption des Sufismus außerhalb muslimisch geprägter Gesellschaften neben „traditionellen“ Sufis, die sich auf die islamische Normenlehre oder diskursive oder rituelle Praktiken der islamischen Tradition beziehen, auch ein Verständnis von Sufismus entwickelt hat, das diesen im Sinne einer universellen Spiritualität jenseits der Grenzen etablierter Religionen und losgelöst von herkömmlichen islamischen Autoritäten interpretiert (Hermansen 2000). Die Arbeit im Forschungscluster sollte sich aber explizit mit „muslimischen“ Sufis befassen. Ich habe das Bestimmungsproblem dadurch zu lösen versucht, dass ich die Abgrenzungsdiskurse zwischen den verschiedenen Gruppen und die Bezugnahme auf den Islam bei dieser Abgrenzung selbst mit zum Gegenstand meiner Untersuchung machte (siehe Dressler 2009 und 2010). Dabei sah ich zunächst all diejenigen Sufis als Teil des islamischen Diskurses an, die sich selbst praktisch oder diskursiv als islamisch positioniert haben und von anderen Musliminnen und Muslimen als Teil dieses

\footnotetext{
6 Verdichtung verstanden als Grad der Systematisierung einer Tradition und Bewusstsein über Zugehörigkeit zu dieser sowie die damit einhergehende Etablierung sozialer Grenzen.

7 Das Projekt war an der Columbia University in New York angesiedelt und finanziert von der Ford Foundation.
} 
Diskurses wahrgenommen wurden. Diejenigen Sufis, die von anderen Musliminnen und Muslimen der Häresie beschuldigt wurden, verstand ich als Teil des islamischen Diskursfeldes, spielt doch die Frage des Aus- beziehungsweise Einschlusses eine entscheidende Rolle bei der Definition von Religionsgrenzen (Bourdieu). So ist es möglich, Unterscheidungsdiskurse, die sich Begriffen wie Häresie (bid' $a)^{8}$ und Unglaube ( $k u f r$ ) bedienen, als Praktiken zu betrachten, über die die Grenzen des islamischen din („Religion“) verhandelt werden (Dressler 2009, 80). Dabei sind weder die Bedeutungsfelder apologetischer islamischer Abgrenzungsbegriffe notwendigerweise statisch, noch die Autoritäten, über die sie Evidenz erhalten. So ist die Bezugnahme auf Hadithkorpora und die diesen zugeschriebene Autorität durchaus umstritten und kann entsprechend zu sehr unterschiedlichen Bestimmungen der Grenzen des islamischen dīn führen.

Auf der Basis dieser Vorannahmen unterschied ich dann zwischen Sufi und non-Sufi Muslims, sowie zwischen Muslim und non-Muslim Sufis. Die Analyse von Dynamiken der Eigen- und Fremdzuschreibungen zum Islam ermöglichte mir, das sufische Feld der Metropole New York als ein muslimisches Diskursfeld zu beschreiben, das sowohl durch innerislamische als auch durch interreligiöse Abgrenzungsdiskurse konstituiert wird. Die Zwänge und Möglichkeiten, die sich aus den sozialen, kulturellen und politischen Dynamiken des nordamerikanischen Raumes in Bezug auf die Artikulation islamischer und sufischer Identitäten ergeben, sind hierbei von grundlegender Bedeutung (Dressler 2009, 77-85). Sie formen den diskursiven Rahmen, innerhalb dessen zunächst zwei Differenzierungsprozesse voneinander unterschieden werden können:

1) In Bezug auf die Unterscheidung zwischen Sufi und non-Sufi Muslims ist die islamische Tradition der Bezugspunkt, vor dessen Hintergrund unterschiedliche Positionen zum Sufismus miteinander um Autorität und Legitimität ringen (Dressler 2010, 436 f.).

2) In Bezug auf die Unterscheidung zwischen Muslim und non-Muslim Sufis markiert der Sufismus die Grenzen des Feldes, innerhalb dessen diese Unterscheidung Evidenz erhält. Aus der Perspektive der Muslim Sufis formuliert das Bekenntnis zum islamischen dìn, im islamischen Diskurs oft mit dem Begriff šarī'a verknüpft, eine eindeutige Grenze zum non-Muslim Sufism (Dressler 2010, 441-445).

8 Generell verwende ich bei islamischen Termini technici die an das Arabische angelehnte gängige Umschrift, außer für türkischsprachige Kontexte, bei denen ich die moderne türkeitürkische Schreibweise übernehme. 
Im von mir untersuchten New Yorker Sufi-Milieu fungierten dīn und šarī $a$ als zentrale Begriffe, über die islamische Orthodoxie formuliert wird. Wie ein Mitglied der New Yorker Ḥaqqanī-Naqšbandiyya erklärte:

„[As for] Sufism in the country [...] people are interested in the Mysticism. [...] They don’t know anything about the šari` $a$. [...] They want the Mysticism. [...] They don’t want the law.” Und er fügte später hinzu: „We're not Mysticism. We're not a different group. We are Islam, we are the mainstream Islam. [...] All these other groups, you know, they're off-base. They're off-key."

Ihr jeweiliges Islamverständnis ist von zentraler Bedeutung für die Selbstpositionierung von Sufi-Gruppen. Während insbesondere muslimische Sufis in der Regel um klare Abgrenzung von nicht-muslimischen Sufis bemüht sind - wie im obigen Beispiel zum Teil auch mit pejorativem Duktus („off-base“, „mysticists“) - zeigt die Beobachterperspektive, dass die hierbei gesteckten Grenzen oft schwerer fassbar sind, als dies von sich klar zum Islam bekennenden Sufis gerne insinuiert wird. Dies ist insbesondere bei denjenigen Sufis der Fall, deren Denken und Sprache stark von mystischen Vorstellungen geprägt ist, die sich bisweilen klaren religiösen Zuordnungen widersetzen und einer Alltagsrationalität widersprüchlich Erscheinendes vereinen können - wie ich unten am Beispiel von Lex Hixon exemplarisch erläutern werde. Zunächst gilt es festzuhalten, dass das diskursive Feld „Sufismus“ konkurrierende Verständnisse von Sufismus beinhaltet, die miteinander in komplexen und nicht nur von religiösen Gesichtspunkten geleiteten einund ausgrenzenden Beziehungen stehen. ${ }^{10}$ Es ist extrem schwierig, überhaupt „den“ emischen Diskurs zum Sufismus abzubilden, da es sich hier um ein heterogenes und dynamisches Diskursfeld handelt, in dem ganz unterschiedliche Positionen miteinander um Deutungshoheit ringen. Deshalb wäre es wenig hilfreich, aus der Dynamik der angedeuteten Grenzziehungsprozesse definitive Trennlinien zwischen Muslim und non-Muslim Sufis ableiten zu wollen.

Eine in Bezug auf unseren Gegenstand äußerst aufschlussreiche objektsprachliche Bestimmung von Religion aus einer mystisch-sufischen Perspektive finden wir bei Lex Hixon, alias Scheich Nur Al Jerrahi (1941-1995). Hixon bietet eine Deutung des Sufismus in seinem Verhältnis zu anderen religiösen Traditionen, die einerseits konventionelle Religionsgrenzen in Frage stellt, andererseits aber den Sufismus aus dem Islam heraus interpretiert und deshalb als Muslim Sufism bezeichnet werden kann - auch wenn manche Musliminnen und Muslime

9 Sufi Focus Group Interview. Muslims in New York City Project, New York: Columbia University, Transkript, 12. September 2000.

10 Ethnische, sprachliche und politische Aspekte können hier ebenso eine Rolle spielen wie die Konkurrenz zwischen Scheichs unterschiedlicher Sufitraditionen. 
die Islamizität Hixons in Frage stellen. Hixon besaß einen Doktortitel in Vergleichender Religionswissenschaft der Columbia University in New York City. Bekannt wurde er jedoch als Dialogiker und religiöser Virtuose, der gleichzeitig verschiedene Religionen studierte und praktizierte. In einem späten autobiografischen Text erklärte er:

„In the Sufi world [...] conventional differences fall away. [...] It doesn't mean that we adopt other religious forms. We stay with the pillars of Islam and with the Sunnah of the Prophet Muhammad, upon him be peace. But we express it in a totally universal manner, in which there are no more barriers or walls.” (Al Jerrahi 1994, 195)

„I can worship along with everyone of every faith. I feel entirely comfortable with that because I don't believe that there are pluralities of faith.” (Al Jerrahi 1994, 198)

Diese Zitate zeigen, dass die mystische Form des Islams in der Interpretation von Lex Hixon eine essentielle Differenz zwischen den Religionen negiert und stattdessen auf eine universale Einheit des Glaubens verweist, während er sich gleichzeitig explizit auf die Bedeutung der „Fünf Säulen“ bezieht und sich damit auch innerhalb des modernen sunnitischen Diskursfeldes verortet. Diesen scheinbaren Widerspruch auflösend, erklärt er an anderem Ort, dass die traditionellen Religionen unverzichtbar seien, um den Weg zur angestrebten mystischen Einheit gehen zu können. In einem Interview von 1991 versicherte er, dass er orthodox in vier verschiedenen Traditionen sei: Dem hinduistischen Ramakrishna-Orden, dem Gelugpa-Orden des tibetischen Buddhismus, der Orthodoxen Kirche des Ostens ${ }^{11}$ und dem Islam. Die Anbindung an verschiedene Religionen wollte er jedoch nicht als Ausdruck einer Verschmelzung dieser Traditionen verstanden wissen. Im Gegenteil, als Traditionalist wandte er sich explizit gegen ein Verständnis von Spiritualität außerhalb der bewährten religiösen Traditionen, ${ }^{12}$ da ein solches zwangsläufig zu einem spirituellen Desaster führen müsse:

„Each of these traditions has its own integrity; they can't be casually mixed together. [...] Sufism or any mystic path should be rooted in one of the noble traditions [...which are] real vehicles for approaching the truth and rejecting the false. I don't think there is any way to operate outside the great vehicles, which are embodied in the cultures that practice them. The great crisis in the modern world is the danger of their disappearance."13

11 Er soll mehrere Jahre am formal der Orthodox Church in America angeschlossenen, aber panorthodox ausgerichteten St. Vladimir‘s Seminary in New York studiert haben. „Lex Hixon, “ Wikipedia, https://en.wikipedia.org/wiki/Lex_Hixon, 18.08.2017.

12 Diese Position erinnert an den Traditionalismus René Guénons. Vgl. Bisson 2007, $31 \mathrm{f}$.

13 Zitiert in Stephanie Golden, „Profile: Lex Hixon,“ Yoga Journal, January/February 1991, 2 f. 
Lex Hixons Verständnis von Sufismus verortet diesen also klar innerhalb des Islams, verknüpft mit einem spezifischen Traditions- und Spiritualitätsverständnis: Die mystische Erfahrung der Glaubenseinheit kann nur über die Anbindung an bewährte Traditionen, „Vehikel“ auf dem spirituellen Weg, erfahren werden. Traditionen sind also klar voneinander getrennt; nur in der mystischen Glaubenserfahrung hebt sich diese Trennung auf. Die in diesem Beispiel offenbare Spannung zwischen statischer Tradition und einer diese Statik voraussetzenden, sie jedoch gleichzeitig transzendierenden Mystik erfordert eine religionswissenschaftliche Metasprache, die solche dynamischen emischen Diskurse abbilden und eine Grundlage für deren Kontextualisierung und Analyse bereitstellen kann, ohne durch die begriffliche Fixierung des Gegenstandes - hier in Bezug auf „Islam“ und „Sufismus“, allgemeiner in Bezug auf „Religion“ - selbst normierend zu wirken. Es geht also zum einen darum, die Mechanismen und Routinen zu hinterfragen, über die wir als Religionsforscherinnen und Religionsforscher bestimmte religiöse Traditionen voneinander abgrenzen. Zum anderen geht es mir auch darum, über einen dynamischen Begriff von religiöser Tradition die Prozesshaftigkeit der Konstituierung religiöser Formationen analytisch besser greifbar zu machen.

\subsection{Grenzziehungen im türkischen Religionsdiskurs}

Mein zweites Beispiel zur Illustration der Herausforderung einer nicht normierenden metasprachlichen Beschreibung des islamischen Diskursfeldes und der dieses konstituierenden Grenzziehungsprozesse stammt aus dem türkischen Religionsdiskurs. 1927 schrieb der nationalistische Journalist und Geschichtslehrer Enver Behnan, der später als Enver Behnan Şapolyo auch zur islamischen Religionsund Sektengeschichte publizieren sollte, in einem reißerischen Artikel über die Kızılbaş, wie die Aleviten damals noch genannt wurden: „Ich frage mich, was das Kızılbaştum ist. Ist es eine Religion ( din), ist es eine religiöse Untergruppe (mezhep), oder ist es etwa ein Sufiorden (tarikat)?“ (Enver Behnan 1927, 12). Die Frage ist natürlich keine zufällige. Im Kontext des kemalistischen Nationalismus der frühen Republik Türkei war die religiöse und ethnische Pluralität des Landes ein Problem. Es ging den Kemalisten, in jungtürkischer Tradition, darum, die Nation zu homogenisieren: sprachlich, kulturell und religiös. Die Aleviten waren besonders von dieser Homogenisierungspolitik betroffen, da sie den meisten Sunniten als Häretiker galten, deren religiöse Andersartigkeit das Potenzial barg, die islamische Einheit der türkischen Nation in Frage zu stellen. Gleichzeitig war die Existenz einer kurdischsprachigen Minderheit unter den Aleviten angesichts der angestrebten Türkifizierung der Kurden äußerst brisant (Dressler 2013a). 
36 Jahre nach dem Erscheinen des Texts von Enver Behnan bediente sich der türkische Religionshistoriker Abdülbaki Gölpınarlı in seiner Diskussion zur Verortung des Alevitentums der exakt selben Begriffe:

„[E]s versteht sich, dass es sich beim Alevitentum nicht um einen Sufiorden (tarikat) handelt. Aber wir können auch nicht sagen, dass es sich bei diesem Weg um eine einwandfreie Rechtsschule (mezhep) handle, da seine Methoden [der Rechtsauslegung] nie bestimmt wurden. Das Alevitentum ist bestenfalls eine primitive Rechtsschule (iptidâî bir mezhep) oder eine primitive Religion (iptidâî bir din).“ (Gölpınarlı 1963, 4) ${ }^{14}$

Enver Behnans Text von 1927 verdeutlichte die Ambiguität des zeitgenössischen Diskurses in Bezug auf die religiöse Positionierung des Alevitentums im Verhältnis zu zentralen Begrifflichkeiten der islamischen Religionstradition. Das Zitat von Gölpınarlı ist Indiz dafür, dass das Problem der Bestimmung des Alevitentums im Verhältnis zum Islam auch in der zweiten Hälfte des 20. Jahrhunderts noch genauso klärungsbedürftig erschien. ${ }^{15}$ Gölpınarlıs religionswissenschaftliche Einordnung des Alevitentums reflektierte allerdings, im Gegensatz zu derjenigen Enver Behnans, nicht mehr die öffentliche Meinung. In den ersten Dekaden der Republik hatte sich nämlich die nationalistische Deutung des Alevitentums als eine „heterodoxe“ Sonderform des Islams türkischer Prägung durchgesetzt (Dressler 2013a). In diesem Kontext erscheint Gölpınarlıs Deutung des Alevitentums als „primitive Religion“, was eine gewisse Unabhängigkeit vom Islam impliziert, wie eine Subversion des nationalistischen Einheitsdiskurses.

Jenseits des politischen Kontexts illustrieren die Aussagen von Enver Behnan und Gölpınarlı die Attraktivität statischer Religionsbegriffe als metasprachliche Ordnungskategorien für komplexe religiöse Felder. Mich interessiert hier die Verwendung von islamischen Begriffen wie mezhep (Arab. madhab), tarikat (Arab. tarīqa) und din (Arab. dìn) zur Normierung des Islams. Innerhalb der sunnitischislamischen Religionskunde sind diese Begriffe recht klar bestimmt: Der Begriff dīn steht im Kern für die absolute göttliche Souveränität ${ }^{16}$ sowie Verpflichtungen des Menschen Gott gegenüber (Haddad 1974). ${ }^{17}$ Die semantische Funktion des Be-

14 Ein evolutionistischer Religionsbegriff, wie hier von Gölpınarlı vorausgesetzt, findet sich auch schon knapp 50 Jahre früher bei Ziya Gökalp sowie bei Mehmed Fuad Köprülü, bei dem Gölpınarlı studierte. Sowohl Gökalp als auch Köprülü waren von Durkheims evolutionistischer Gesellschaftstheorie beeinflusst (Dressler 2013a, 187 f.).

15 Bis heute hat sich das nicht wesentlich geändert (siehe Dressler 2013b).

16 Emblematisch verkörpert im koranischen Begriff yawm al-dīn, der „Tag des Gerichts“, beziehungsweise ,Jüngster Tag“.

17 Die Etymologie des arabischen Wortes dīn ist umstritten. In der wissenschaftlichen Literatur werden arabische, hebräische, aramäische sowie persische Ableitungen diskutiert (Haußig 1999, $216 \mathrm{f}$.$) .$ 
griffs ist somit klar normativ und kann sich auf prinzipiell alle Lebensbereiche beziehen (Schielke 2010, 3). Dabei wird auch den Juden und Christen als „Schriftbesitzern“ (ahl al-kitāb) ein dīn zugesprochen, auch wenn deren Umgang mit ihrem dīn im Koran kritisiert wird und wenn das islamische Verständnis von dīn als gottgefälliges menschliches Tun als überlegen verstanden wird (Gardet 2017; Tümer 1994, 313). Der Begriff madhab bezeichnet im weiteren Sinne eine Art des Denkens oder eine Überzeugung, spezifischer steht er für die allgemein anerkannten Auslegungstraditionen der islamischen Normenlehre (im Deutschen als ,islamische Rechtsschulen“ bekannt) (Ed. [1986]). Der Begriff tarīqa schließlich wird für die innerhalb des Sufismus ausgebildeten Ordenstraditionen verwendet.

Nun werden diese islamischen Begrifflichkeiten im Türkischen auch dazu benutzt, mittels Analogie Gruppierungen, die außerhalb des islamischen Diskurses stehen, zu kategorisieren. Ähnlich wie christliche Religionskategorien durch den Weltreligionendiskurs globalisiert wurden und Termini mit spezifisch christlichen Wurzeln wie Religion, Theologie, Konfession, Denomination, Ortho- und Heterodoxie oder Häresie als trans-traditionale Ordnungs- und Analysebegriffe verwendet werden, wird auch hier, ausgehend von einem islamischen Religionsverständnis, ein universalistischer Religionsdiskurs gebildet. So hat der Begriff dìn neben seiner exklusiven Verwendung für den Islam im Sinne von ,die Religion“ (al-dīn) schon im Türkischen des späten Osmanischen Reiches auch die weitere Bedeutung von Religion im Sinne eines universellen Gattungsbegriffs angenommen, ${ }^{18}$ mit dem dann auch Traditionen außerhalb des traditionellen islamischen Verständnisses von dīn/Religion wie der Buddhismus oder eben auch das Alevitentum bezeichnen werden konnten. Die obigen Beispiele von Enver Behnan und Gölpınarlı illustrieren, dass sich diese generalisierende Verwendung in der Türkei neben der Persistenz der nach wie vor dominanten islamischen Bedeutungen der besagten Termini etabliert hat. Die staatliche Türkische Sprachgesellschaft (Türk Dil Kurumu) definiert din im generalisierten Sinne als ,eine gesellschaftliche Institution und Religiosität (toplumsal bir kurum, diyanet), die den Glauben und die Verehrung Gottes, übernatürlicher Kräfte und verschiedener heiliger Wesenheiten systematisiert. " ${ }^{19}$ Differenzierter ist der Eintrag din in der Diyanet İslam Ansiklopedisi, der wichtigsten türkischsprachigen islamisch-theologischen Enzyklopädie. Nach einer Diskussion der Etymologie und der koranischen Bedeutungen des Begriffs werden vermeintliche Äquivalente von din in anderen

18 Unter dem Eintrag din verweist das Wörterbuch Kamus-1 Osmani auf die Existenz ,verschiedener Religionen (edyan) und religiöser Untergruppen (mezahib)” (Salahi 1895-1904, Eintrag „din“). Das Kamus-1 Türki (1899) bestätigt dies: ,es gibt ziemlich viele [unterschiedliche] Religionen (edyan) in der Welt“ (Sami 2017, 745).

19 Türk Dil Kurumu, Güncel Türkçe Sözlük, http://www.tdk.gov.tr/, 20.02.2018. 
Religionen untersucht (z.B. religio und dharma), wobei jedoch die Einzigartigkeit des Begriffs din betont wird. Nichtsdestotrotz werden auch hier die verglichenen Traditionen selbstredend als dinler („Religionen“) bezeichnet (Tümer 1994, 314). Din steht also auch hier sowohl für das Allgemeine (Weltreligionen) als auch das Besondere (der islamische din). Einen wesentlich restriktiveren Begriff von din offeriert Abdurrahman Dilipak, einer der einflussreichsten islamistischen Journalisten und Intellektuellen der Türkei. In einem rezenten Zeitungsartikel beklagt er, dass oft als din wahrgenommen werde, was eigentlich der Tradition oder der Kultur zuzurechnen sei. Diese Vermischung sei im Christentum und Judentum noch stärker ausgeprägt als im Islam. Dilipak erklärt dies damit, dass „[v]or allem das Christentum kein din ist, sondern religio. Es hat keinen göttlichen Kern. “20 Für Dilipak ist das Herz des Islams sein din, was jedoch nicht mit religio gleichzusetzen sei. Religio ist für ihn Ausdruck der Verfälschung des göttlichen din, praktisch ausgedrückt in seiner Vermischung mit Kultur.

Analog zu der oben beschriebenen Bedeutungsdualität von din werden die Begriffe mezhep und tarikat, neben ihren spezifisch islamischen Bedeutungen, im türkischen Religionsdiskurs zusätzlich auch dazu verwendet, aus dem globalisierten Religionsdiskurs bekannte Konzepte christlicher Provenienz wie Sekte, Denomination, Konfession oder Orden auszudrücken. So wird im türkisch-türkischen Lexikon der Türkischen Sprachgesellschaft der Terminus mezhep ohne direkten Islambezug beschrieben als ,jeder Zweig einer Religion, der aufgrund von Differenzen in Bezug auf Ansicht, Auslegung und Verständnis entstanden ist.“21 Und tarikat wird, ebenfalls ohne expliziten Islambezug, bestimmt als ,alle sich aus Unterschieden in der Interpretation und in der Praxis innerhalb derselben Religion ergebenden und in Bezug auf manche Prinzipien sich voneinander unterscheidenden Wege zum Erreichen und Erkennen Gottes.“22 Mithin werden hier islamische Begriffe in einer Weise generalisiert, die Kompatibilität mit dem globalisierten Weltreligionendiskurs herstellt. Den türkischen Religionsdiskurs könnte man dann als Produkt einer „Globalarabisierung“ basierend auf „globallateinischem“ Vorbild verstehen. ${ }^{23}$ Jedoch hat diese Tendenz zur Globalarabisierung Grenzen. Die Einträge mezhep und tarikat in der Diyanet İslam Ansiklopedisi bleiben, im Gegensatz zu denjenigen im Wörterbuch der Türkischen Sprachgesellschaft, ganz im islamischen Kontext und zeigen keine universalisierende Tendenz (siehe Üzüm 2004; Öngören 2011).

20 Abdurrahman Dilipak, „Bunlar İsevi de değil!“, Yeni Akit, 08.10.2017, http://www.yeniakit.co m.tr/yazarlar/abdurrahman-dilipak/otekilere-21320.html.

21 Türk Dil Kurumu, Güncel Türkçe Sözlük, http://www.tdk.gov.tr, 07.02.2015.

22 Türk Dil Kurumu, Güncel Türkçe Sözlük, http://www.tdk.gov.tr, 07.02.2015.

23 Angelehnt an Derridas (2002, 66 f.) Begriff der Globallatinisierung. 
Die Religionisierung des Islams in der Moderne bedeutet nicht, dass der vormoderne islamische Begriff von din keine Berührungspunkte und semantische Affinitäten mit dem westlichen Religionsbegriff hätte. ${ }^{24}$ Wie bereits Wilfred Cantwell Smith betont hat, sind schon im Koran traditionsübergreifende und komparativistische Tendenzen angelegt (Smith 1991, 81 f.; Haußig 1999, insb. 227-234). Auch für spätere Zeiten finden sich immer wieder Belege dafür, dass der Begriff dīn, neben seinen spezifisch islamischen Bedeutungen, auch als Basis für einen - auf islamischen Semantiken beruhenden - Religionsvergleich benutzt wurde (Riesebrodt 2004, 132 f.; Karamustafa 2017, 166 f.). Im modernen türkischen Religionsdiskurs ist diese Globalisierung islamischer Religionssemantik recht weit fortgeschritten, wird jedoch nicht reflektiert. Dieser globalisierte Diskurs über Religion operiert zudem innerhalb eines säkularistischen Verständnisses der Differenzierung sozialer Sphären. So sehen sich Aleviten oft mit der Frage konfrontiert, ob ihre Tradition nicht weniger eine Religion im engeren Sinne als vielmehr eine spezifisch anatolische Philosophie oder Lebensweise, eine anatolische Kultur, eine Ethnie oder eine politische Haltung/Ideologie sei. Die Frage impliziert einen statischen Religionsbegriff, der, an der dem modernen Religionsbegriff zugrundeliegenden Differenzierungsthese angelehnt, klar zwischen Religion und anderen Werte- und Lebensbereichen unterscheidet (Dressler 2008).

\section{Metasprachliche Reflexion}

In Anbetracht des dynamischen Charakters innerreligiöser Differenzierungsprozesse stellt sich die Frage, wie man diesen metasprachlich adäquat abbilden kann. Sowohl das von den türkischen Autoren Enver Behnan und Gölpınarlı aufgeworfene Problem der religionsbegrifflichen Kategorisierung des Alevitentums als auch das diffizile Verhältnis von Sufismus zu Islam und die Frage, wie man zwischen islamischem und nicht-islamischem Sufismus analytisch unterscheiden kann, implizieren klar umrissene Grenzen des Islams. Ein konventioneller, statischer Religionsbegriff offeriert im Wesentlichen drei Möglichkeiten der Positionierung von marginalen Gruppierungen im Verhältnis zu einem hegemonialen Religionsdiskurs: innerhalb, außerhalb oder liminal, im Grenzbereich zwischen Innen und Außen. Da solche Positionierungen, die im religionswissenschaftlichen Diskurs oft mit Hilfe von Begriffen wie Heterodoxie und/oder Synkretismus

24 Zum Begriff der Religionisierung siehe Dreßler, Markus. "Modes of Religionization: A Constructivist Approach to Secularity." Working Paper Series of the HCAS "Multiple Secularities - Beyond the West, Beyond Modernities“ 7, Leipzig, February 2019. Online: https://www.multiple-secularities.de/media/wps7_dressler_religionization.pdf. 
beschrieben werden, von einer ständigen Abgrenzung gegenüber objektivierten Bezugsgrößen („Orthodoxien“) diskursiv abhängig sind, bleibt ihr analytischer Wert jedoch begrenzt. ${ }^{25}$ Es geht mir also um ein heuristisches Modell, mit Hilfe dessen die statische Beschreibungslogik (innerhalb/außerhalb/liminal) überwunden werden kann.

\subsection{Stammbaummodell versus Wellenmodell}

Das statische Religionsmodell und die von ihm implizierten klaren Grenzen zwischen Religionen können exemplarisch am Stammbaummodell von Religion veranschaulicht werden. ${ }^{26}$ Dieses Modell nimmt an, dass jede Religion historisch eindeutig verortbare Ursprünge hat (Boyarin 1999, 9; 2004, 18 f.). Die Annahme der Freilegbarkeit ihrer historischen Wurzeln suggeriert gleichzeitig, dass Religionen klar von anderen Religionen sowie von nichtreligiösen Phänomenen unterschieden werden können. Beziehungen zwischen Religionen werden über die Bestimmung von Ursprüngen, Einflüssen, Schismen und Verschmelzungen geklärt, „Religionen“ dadurch analytisch zerlegt und systematisch geordnet. ${ }^{27}$ Das Problem dieses Modells ist sein impliziter Essenzialismus in Bezug auf die Inhalte und Grenzen religiöser Traditionen. Nicht von ungefähr hat das Stammbaummodell von Religion eine semantische Affinität zum Weltreligionenmodell (siehe Masuzawa 2005). Beide Modelle vertragen sich gut mit der modernen Differenzierungsthese, die den Fokus auf die Unterscheidung der Religion von anderen sozialen Räumen legt. ${ }^{28}$ Dadurch läuft man aber Gefahr, der Hybridität religiöser Traditionen im Verhältnis zueinander sowie der faktischen Verquickung religiöser und nicht-religiöser Praktiken und Diskurse auch in der Moderne zu wenig Aufmerksamkeit zuzuwenden oder gar zu einer Reifikation des Religiösen (Smith 1991) beizutragen. Diese Logik kann, wie das türkische Beispiel zeigt, durch nationalistische Diskurse, denen daran gelegen ist, Ursprünge und Grenzen in Bezug auf ethnische, sprachliche, religiöse und im weiteren Sinne kulturelle Grenzen mög-

25 Das Problem modernistischer Konzeptualisierungen inter- und innerreligiöser Andersartigkeit am Beispiel des Diskurses über die Kızılbaş-Aleviten habe ich andernorts ausführlich diskutiert (Dressler 2013a, insbesondere Kap. 5).

26 Für eine rezente religionswissenschaftliche Arbeit mit dem Stammbaummodell, in dem auch klassische Ansätze diskutiert werden, siehe Wunn 2018.

27 Vgl. die Kritik von Deleuze und Guattari (1977, 8 f.) am „Wurzelbaum“ und dem ihm zugrundliegenden binären Denken.

28 Für eine Beschreibung der Differenzierungsthese siehe z.B. Casanova 1994, 20-25; vgl. Luhmann 2013. 
lichst harmonisch aufeinander abzustimmen und Widersprüche zu glätten, noch zusätzlich verstärkt werden. ${ }^{29}$

Perspektiven auf das Alevitentum vom Blickwinkel des Stammbaummodells erkennen in diesem eine Vermischung schiitisch-islamischer Elemente mit Praktiken und Vorstellungen nicht-islamischer Traditionen (je nach Lesart werden hier vorislamische türkische oder kurdische beziehungsweise persische, aber auch christliche und/oder anatolische Einflüsse stark gemacht). Die herkömmliche orientalistische Forschungstradition deutet solche Prozesse mit analytischen und als wertneutral vorgestellten Begriffen wie Synkretismus und Heterodoxie. In der Perspektive der sunnitischen Gelehrtentradition sowie im Alltagsverständnis vieler Laien überschneiden sich jedoch die semantischen Felder der Begriffe Häresie und Heterodoxie weitgehend (siehe Dressler 2013a, Kap. 5).

Als Alternative zum statischen Stammbaummodell schlägt der Spätantikeforscher Daniel Boyarin einen dynamischen Religionsbegriff in Anlehnung an das sprachwissenschaftliche Wellenmodell vor:

\begin{abstract}
„Wave theory posits that linguistic similarity is not necessarily the product of a common origin but may be the product of convergence of different dialects spoken in contiguous areas, dialects that are, moreover, not strictly bounded and differentiated from each other but instead shade one into the other. Innovations at any one point spread like the waves created when a stone is thrown into a pond, intersecting with other such waves produced in other places and leading to the currently observed patterns of differentiation and similarity." (Boyarin 2004, 18)
\end{abstract}

Wo die Grenzen einer Sprache gesetzt werden, kann genauso wenig sprachimmanent geklärt werden wie die Frage, ob man eine bestimmte Sprachstufe besser als Sprache oder als Dialekt kategorisieren sollte. Sprachgrenzen sind immer sozial konstruiert, Produkt eines Normierungsprozesses innerhalb eines sprachpolitischen Feldes, das durch die Machtpositionen und Interessen verschiedener Institutionen und Akteure strukturiert wird. Man kann dieses Modell auch auf Religion übertragen. Religionen erscheinen dann als das Resultat komplexer historischer Normierungen von als „religiös“ gedeuteten Praktiken.

Das von Boyarin offerierte Wellenmodell steht im Widerspruch zur statischen Deutung von Religionen als historische Einheiten mit prinzipiell bestimmbaren Ursprüngen und Grenzen. ${ }^{30}$ Klarheit in Bezug auf Ursprünge und Grenzen ergibt

29 Für das Beispiel der nationalistisch inspirierten Türkisierung und religiösen Assimilation des Alevitentums siehe Dressler 2013a. Ein anschauliches Beispiel bietet auch Adam Beckers rezente Rekonstruktion der Genese des assyrischen Nationalismus (Becker 2015, insbesondere Kap. 8).

30 Für einen einflussreichen Beitrag zur religionswissenschaftlichen Theoriebildung auf der Basis eines Fluidität betonenden Religionsbegriffs siehe Tweed 2006. 
sich in der Regel nicht aus historischen Tatsachen selber, sondern ist von etablierten Deutungsmustern abhängig. Die soziale Konstruktion von Religion(en) steht also in einer Wechselbeziehung mit sozialen und historischen Strukturen (Dressler 2018). Innerhalb religiöser Felder stehen normierte sowie gleichzeitig normierende Praxis, Dogmatik und Apologetik in komplexen Verhältnissen zu dem, was sie zu korrigieren beziehungsweise auszuschließen suchen: religiöse Indifferenz sowie Häresie beziehungsweise Pluralität und Mehrdeutigkeit bestimmter Vorstellungen und Praktiken. Hinzu kommt, dass religiöse Traditionen nicht notwendigerweise klar voneinander abgetrennt und unterscheidbar sind, sondern oft in vielfältigem Kontakt und Austausch (von Dialog bis Abgrenzung) stehen. Man kann dies mit der Metapher der „Kontaktzone“ (Mary Louise Pratt) beschreiben, die auf den Ort verweist, an dem Transkulturation stattfindet und wo verschiedene soziokulturelle Formationen sich in einer dynamischen „Konversation“ (Boyarin 1999, 11-16) miteinander befinden. Während in der alltagsweltlichen sozialen Konstruktion von Religion, insbesondere, aber nicht ausschließlich durch Akteure, die sich selbst einer Religion zuordnen, Religionsgrenzen meist „wirklich“ sind, beinhalten aus religionsgeschichtlicher Perspektive religiöse Traditionen immer auch Aspekte von Hybridität. Grenzen zwischen religiösen Traditionen haben aus dieser Perspektive keine a priori gegebene Faktizität, sondern werden erst in komplexen Prozessen im Kontext divergierender Interessen und Machtverhältnisse zu sozialen Fakten verdichtet (Boyarin 2004, 18 f.). Religiöse Traditionen können mithin als Produkt der Überlagerung und Verdichtung von Welleneffekten mit diskursiv als „religiös“ markierter Ladung verstanden werden. ${ }^{31}$

31 Abseits des Religionskontexts finden sich weitere interessante Ansätze, Differenz als dynamischen Prozess und nicht als statischen Zustand zu beschreiben. Ein Beispiel ist die Metapher vom Rhizom, die Deleuze und Guittari in Abgrenzung zum binären Wurzelbaum-Modell gebildet haben. Das Rhizom ist charakterisiert durch das „Prinzip der Konnexion und der Heterogenität. Jeder beliebige Punkt eines Rhizoms kann und muß mit jedem anderen verbunden werden. Ganz anders dagegen der Baum oder die Wurzel, wo ein Punkt und eine Ordnung festgesetzt werden“ (Deleuze und Guittari 1977, 11). Im Unterschied zu der biologistischen Metaphorik des Rhizoms offeriert der Begriff partial connections, der von Marilyn Strathern in Konversation mit Donna Haraway und James Gleick entwickelt wurde, einen mathematisch inspirierten Zugang: „The notion of partial connections offers [...] the possibility of conceptualizing entities (or collectives) with relations integrally implied, thus disrupting them as units; emerging from the relation, entities are intra-related [...] instead of being inter-related [...] Instead of plurality (a feature premised on units), the mathematical image congenial to partial connections is that of fractals: they offer the possibility of describing irregular bodies that escape Euclidian geometrical measurements because their borders allow other bodies in - without, however, touching each other everywhere“ (Cadena 2015, 32). Cadena $(2015,33)$ spricht in diesem Zusammenhang auch von „kaleidoscopic simultaneity of similarity and difference“. 


\subsection{Religion und religiöse Tradition}

Ich schlage im Folgenden vor, einen weiteren Begriff von „religiöser Tradition“ von einem engeren Begriff von „Religion“ zu unterscheiden. Als Religion verstehe ich mithin religiöse Traditionen, die einen normativen Diskurs bezüglich ihrer eigenen Grenzen aufweisen. Demgegenüber soll der vorgeschlagene weitere Begriff von religiöser Tradition auch soziokulturelle Formationen miteinschließen, die keine autoritativen Diskurse über ihre eigenen Grenzen entwickelt haben, beziehungsweise die sich im Prozess der Entwicklung solcher Grenzen befinden. Diese Unterscheidung ist eine heuristische, bei der es darum geht, auch niederschwellige Prozesse der Verdichtung von sozialen Strukturen und Wissenskomplexen (Symbolebene, Sprache, Praktiken) analytisch fassbar zu machen. Die Grenze zwischen religiöser Tradition und Religion wird diesem Vorschlag zufolge vom Grad der Etablierung eines Diskurses der normativen Selbstvergewisserung abhängig gemacht. Mithin stehen diskursive Grenzziehungsprozesse zwischen dem Eigenen und dem Anderen (we-group formation) und deren religionspolitische Kontexte (im Sinne des bourdieuschen Feldbegriffs) im Zentrum der Aufmerksamkeit. In Abgrenzung von einem Traditionsbegriff der sich, wie bei Asad in AoI, an den diskursiv normierten Autoritäten („Orthodoxie“) einer religiösen Tradition ausrichtet, will der hier entwickelte Begriff der religiösen Tradition es über die Unterscheidung unterschiedlicher Grade von Verdichtung von Religionsbildungsprozessen ermöglichen, noch stärker die normierenden Prozesse selbst in den Blick zu nehmen.

Der hier vorgestellte Begriff von Tradition ist zugegebenermaßen etwas unkonventionell und unterscheidet sich von in den Geisteswissenschaften gängigen Traditionsbegriffen wie zum Beispiel dem konstruktivistischen Ansatz, der von Hobsbawm und Ranger in Invention of Tradition (1983) zugrunde gelegt wurde. In der Religionswissenschaft verwandte schon Wilfred Cantwell Smith einen konstruktivistischen Begriff von Tradition: „A religious tradition, then, is the historical construct, in continuous and continuing construction, of those who participate in it“ (Smith 1991, 165). Neben konstruktivistischen Zugängen zum Traditionsbegriff finden sich in der Religionswissenschaft nach wie vor auch Begriffe von Tradition im Sinne historischer Strukturen. Martin Riesebrodt zum Beispiel unterschied religiöse Traditionen (die sich ,auf die historische Kontinuität von Symbolsystemen“ beziehen) von Religionen (,konkrete Systeme von Praktiken“) (Riesebrodt 2007, 13). Gregory Grieve und Richard Weiss haben indes vorgeschlagen, den konstruktivistischen Ansatz, von dem sie zunächst ausgehen, mit einem strukturalistischen Ansatz zu verknüpfen. Sie argumentieren zunächst: „Formulations of traditions and community affirm a synchronic bond between actors and extend that bond into the past, into a diachronic community“ (Grieve und Weiss 
2005, 3). Dem fügen sie jedoch hinzu: „[S]cholars too often underestimate the conservative forces of history, tradition in Marx's and Weber's sense as structures formed through history that limit the range of possible action" (Grieve und Weiss 2005, 4 f.).

Im Unterschied zum konstruktivistischen Zugang möchte auch ich auf einen analytischen Begriff von Tradition nicht verzichten. Im Gegensatz zu einem strukturalistischen Begriff von Tradition, der schon einen fortgeschrittenen Grad sozialer Objektivierung voraussetzt, schlage ich jedoch vor, mit dem Begriff der religiösen Tradition schon niederschwellige Kontinuitäten sozialer und diskursiver Praxis abzubilden. ${ }^{32}$ Damit unterscheidet sich der hier vorgeschlagene Begriff von Tradition auch vom Traditionsbegriff Asads, der zu Beginn dieses Aufsatzes diskutiert wurde. Mithin sollen Prozesse der Verdichtung sozialer Praktiken und gemeinschaftsbildender Diskurse von der (religiösen) Tradition zur Religion ins Zentrum der Aufmerksamkeit gestellt werden. Der heuristische Nutzen der Unterscheidung von religiöser Tradition und Religion liegt im Fokus auf die Prozesshaftigkeit der Religionsformierung beziehungsweise Religionisierung. ${ }^{33}$ Es soll dabei keine evolutionistische Kausalität insinuiert werden. Wenn die Trägerinnen und Träger einer religiösen Tradition beginnen, sich so stark über sich selbst zu vergewissern, dass sie über ein klares Bewusstsein bezüglich der Grenzen der eigenen zu anderen Traditionen verfügen und den Inhalten ihrer Tradition eine systematische Struktur verleihen, die sich auch in sozialen Grenzziehungspraktiken niederschlägt, nenne ich das im Folgenden Religion.

Die Differenzierung zwischen Religion (als soziokulturelle Formation mit klaren Grenzen und systematisiertem Selbstverständnis) und religiöser Tradition hat den Vorteil, dass damit die Dynamiken emischer Aushandlungsprozesse besser erfasst werden können. Sowohl der traditionalistische Sufismus von Lex Hixon als auch die Aleviten können als sich im Prozess der Verdichtung befindliche religiöse Traditionen verstanden werden, wobei dieser Prozess bei letzteren relativ weiter fortgeschritten ist. Im Unterschied zur Religion kann der Begriff der religiö-

32 Auch Reinhard Schulze (2015, pas.) verwendet einen Begriff von Tradition, der einen geringeren Grad der Institutionalisierung voraussetzt als derjenige der Religion. Er versteht Tradition dabei als „Begriff, um das diachrone Gefüge eines Konsenses über ein formales Repertoire symbolischer Repräsentationen zur Etablierung von Deutungswelten zu bezeichnen. [...] Tradition soll hier verstanden werden als das Repertoire von konventionalisierten Trademen“ (Schulze 2015, 151).

33 Als semantische Klammer für die Unterscheidung zwischen religiöser Tradition und Religion verstehe ich hier als „religiös“ Dimensionen des Fühlens, Denkens und Tuns, die als über die Begrenztheit menschlicher Existenz hinausweisend interpretiert werden, sowie diskursive Handlungen und materielle Strukturen, die auf solche Dimensionen Bezug nehmen, beziehungsweise mit ihnen in Bezug gesetzt werden. 
sen Tradition also auch für soziokulturelle Formationen verwendet werden, deren Grenzen zu anderen (religiösen) Traditionen nicht klar definiert sind und bei denen es Überschneidungen mit anderen religiösen Traditionen beziehungsweise mit nichtreligiösen Wissenskomplexen gibt. Er erlaubt es zudem, religiöse Traditionen nicht nur oder in erster Linie im Nebeneinander oder Nacheinander zu denken (Stammbaummodell), sondern auch im Mit-, Zwischen- und Durcheinander als sich synchron sowie diachron potenziell überlagernde Wissenskulturen von unterschiedlichem Verdichtungsgrad - Verdichtungsgrad verstanden als Indikator der Systematisierung eines Traditionskomplexes, des Bewusstseins über die Zugehörigkeit zu diesem Komplex und der Etablierung entsprechender sozialer Grenzen.

Volkhard Krech spricht auch von Verdichtung im Kontext von Traditionsbildung, und zwar im Sinne eines ,process of densification of cultural goods [that] relates to the intensification of content, the density of interaction between the carriers of tradition and the duration of interaction“ (Krech 2012, 41). Das hier vorgeschlagene Verständnis von Verdichtung ähnelt demjenigen Krechs, mit dem Unterschied, dass der von mir vorgeschlagene Traditionsbegriff einen niedrigeren Grad der Verdichtung impliziert und mit Verdichtung somit das Augenmerk auf den Übergang von der (religiösen) Tradition zur Religion gelegt wird. Krech hingegen sieht Traditionen als stärker verdichtete soziale Formationen an.

Es sollte klar sein, dass die vorgeschlagene Unterscheidung zwischen religiöser Tradition und Religion als ein heuristisch begründetes Konstrukt religionswissenschaftlicher Konzeptarbeit sich nicht notwendigerweise damit deckt, wie die entsprechenden Erfahrungen, sozialen Komplexe und Wissenskonstrukte auf der Akteursebene empfunden und objektsprachlich interpretiert werden. Der vorgeschlagene Begriff von religiöser Tradition kann in manchen Fällen in starker Spannung zu objektsprachlichen Diskursen stehen. So können auch soziokulturelle Formationen innerhalb herkömmlicher Religionen als im beschriebenen Sinne zu Religion verdichtete religiöse Traditionen verstanden werden - wenn sie einen entsprechend hohen Verdichtungsgrad aufweisen, wie z.B. Schia und Sunna im islamischen Traditionskontext. ${ }^{34}$ Der vorgeschlagene Traditionsbegriff ist gleichwohl nicht losgelöst von der sozialen Realität, sondern als analytische Reflexion über diese zu verstehen. Die Frage, ob Sunna, Schia oder auch Alevitentum als eigenständige Religionen anzusehen sind oder man die Religionsgrenze weiter fasst, um eine übergeordnete islamische Religionskategorie aufrechtzuer-

34 Für den christlichen Traditionskomplex könnte man ebenso von einem Cluster unterschiedlicher Religionen innerhalb der übergeordneten Kategorie Christentum sprechen. 
halten (und das Verhältnis der genannten Gemeinschaften im Sinne eines Wettbewerbs um die Diskurshoheit bei der Bestimmung islamischer Normativität betrachtet), ist zudem keine bloß akademische. In vielen muslimischen sowie nichtmuslimischen Kontexten hat sie realpolitische Implikationen, die im Kontext spezifischer religionspolitischer Auseinandersetzungen verhandelt werden.

Die Unterscheidung zwischen Religion und religiöser Tradition kann auch zur Untersuchung des Verhältnisses einander nahestehender, aber als eigenständig wahrgenommener religiöser Traditionen herangezogen werden. Neuere historische Arbeiten betonen, dass Religionen wie zum Beispiel Islam, Christentum und Judentum (teilweise auch der Zoroastrismus) oft stärker von vielfältigen Kontakten untereinander und semantischen Überlagerungen geprägt waren als gemeinhin angenommen. ${ }^{35}$ Während der Religionsbegriff ein hohes Maß an Autonomie suggeriert, erlaubt es der Begriff der religiösen Tradition, auch die (synchronen und diachronen) Überschneidungen zwischen diesen sowie mit ihnen verwandten religiösen Traditionen zu beleuchten. Er ermöglicht es, religiöse Konstellationen mit niedrigem Verdichtungsgrad $\mathrm{zu}$ fassen, ohne diese voreilig zu religionisieren, ohne also Grade der Verdichtung zu unterstellen, wo diese empirisch noch nicht nachgewiesen werden können. Aus dieser Sicht kann man zum Beispiel der unter anderem von Boyarin (2004) aufgeworfenen Frage, ab wann man das spätantike Judentum als Religion ansehen kann, mit dem Vorschlag entgegnen, dieses zunächst als religiöse Tradition zu verstehen, teilhabend an verschiedenen religiösen Traditionen unterschiedlichen Verdichtungsgrades. Das ermöglicht dann auch einen analytischen Blick auf die Frage, wann und wie das Judentum religionisiert wurde. Die Herausbildung einer jüdischen Dogmatik und einer jüdischen Orthodoxie (d.h., die Verdichtung des Judentums zur Religion) kann somit im Kontext des spätantiken Religionsfeldes als dynamischer Prozess beschrieben werden.

Der Begriff der religiösen Tradition hilft auch, von der Moderne her entwickelte und in vormoderne Kontexte rückprojizierte Fragen nach Ursprüngen, Kontinuitäten und Religionsgrenzen zu problematisieren, ohne dabei Rekurs auf problematische Ordnungsbegriffe wie z.B. Synkretismus, Heterodoxie oder Häresie zu nehmen, die zum Teil selbst auf religionsimmanenten Semantiken beruhen

35 Boyarin 2004 sowie Becker und Reed 2003 zeigen, dass die Ausdifferenzierung von Christentum und Judentum in der Spätantike ein längerer und komplexerer Prozess war als dies herkömmliche Narrative der Entstehung des Christentums als eigenständiger Religion nahelegen. Zum Islam als spätantiker Religion, die sich in engem Kontakt zu Christentum und Judentum herausbildete, siehe Neuwirth 2010, Hoyland 2012 und 2015, Hughes 2017; zu den diversen Abgrenzungsstrategien, die Christen seit dem frühen Mittelalter gegenüber dem Islam entwickelten, siehe Murre-van den Berg, Ginkel und van Lint 2005. 
und zur diskursiven Essenzialisierung von Religionen beitragen. Ein dynamischer Begriff von religiöser Tradition sollte einen Beitrag dazu leisten, eine analytische Metasprache zu entwickeln, die es erlaubt, Prozesse von Diffusion, Absorption, Adoption, Abgrenzung, Verdichtung, Konsolidierung sowie Auflösung innerhalb und zwischen religiösen Wissenskomplexen in ihren materiellen, kognitiven, affektiven und sozialen Manifestationen sowie politischen und sozioökonomischen Kontexten synchron und diachron $\mathrm{zu}$ beschreiben und $\mathrm{zu}$ analysieren (siehe auch Wick 2012; Krech 2012).

Die Arbeiten Boyarins sind ein gutes Beispiel für die Verwendung eines dynamischen Religionsbegriffs. Boyarin zeigt an der graduellen Ausdifferenzierung von Christentum und Judentum in eigenständige Religionen (die im zweiten Jahrhundert beginnt und erst in der Spätantike, irgendwann im 4. oder 5. Jahrhundert, als abgeschlossen angesehen werden kann), dass der Diskurs über Orthodoxie und Häresie eine zentrale Arena der Herausbildung von Judentum und Christentum bildete. Die Etablierung eines apologetischen Diskurses durch christliche Autoren des zweiten Jahrhunderts und parallel dazu die Entwicklung eines vergleichbaren rabbinischen Diskurses haben Boyarin zufolge wesentlich zur Ausbildung von christlicher, respektive jüdischer Orthodoxie und den damit einhergehenden Grenzziehungen beigetragen. Die Trennung zwischen christlicher und rabbinisch-jüdischer Tradition veranschaulicht, wie ein Binnendiskurs über die Frage korrekter Glaubenslehre und -praktiken sowie über die Basis der sozialen Grenzen der Gemeinschaft zu einer Trennung in zwei sich als eigenständige Traditionen verstehende Gruppen führen kann. Mit dem in diesem Kontext eingeführten Religionsbegriff setzten sich Christen vom Judentum als einer sich stark ethnisch definierenden Gemeinschaft auf qualitativ neue Weise ab:

„Groups that are differentiated in various ways by class, ethnicity, and other forms of social differentiation become transformed into ,religions' in large part, I would suggest, through discourses of orthodoxy/heresy. Early Christian heresiology, whatever else it is, is largely the work of those who wished to eradicate the fuzziness of the borders, semantic and social, between Jews and Christians and thus produce Judaism and Christianity as fully separate (and opposed) entities-as religions, at least in the eye of Christianity.” (Boyarin 2004, 2)

Der von Boyarin beschriebene Prozess markiert in der hier vorgeschlagenen Terminologie die Transformation von religiösen Traditionen in Religionen, das heißt die Religionisierung von Christentum und Judentum. Ich denke, es lohnt, sich als Religionswissenschaftler bisweilen daran zu erinnern, dass die Herausbildung des Christentums als eigenständige Tradition (als Religion im Sinne einer nach innen und außen klar bestimmten, verdichteten religiösen Tradition) einen wichtigen Meilenstein in der Genealogie des modernen Religionsbegriffs markierte. Boyarin zufolge wurde der Religionsbegriff im spätantiken Christentum umge- 
deutet im Sinne einer Bekenntnisgemeinschaft jenseits von ethnischen Grenzen und Verbindlichkeiten. ${ }^{36}$

Versteht man religiöse Traditionen als sich synchron und diachron überlagernde Wissenskulturen oder als Konversationen zwischen religiösen Wissenssträngen, dann erscheinen Begriffe wie Grenze oder Schwelle, die auf eindeutige Entitäten verweisen, als normative Setzungen innerhalb dynamischer sozialer Felder. Allerdings besitzen solche Grenzen in der sozialen Wirklichkeit eine unbestreitbare Evidenz und verweisen auf stark verdichtete religiöse Traditionen: auf Religionen im Sinne klar definierter sozialer Formationen.

Man kann gegen die von mir vorgeschlagene heuristische Unterscheidung zwischen religiöser Tradition und Religion einwenden, dass diese eine Setzung von außen ist, die sich in vielen Fällen kaum mit der Selbstwahrnehmung religiöser Gruppen deckt. ${ }^{37}$ Mir geht es jedoch nicht darum, eine normierende Begrifflichkeit einzuführen, mittels derer dann ihrerseits wieder kategoriale Grenzen zwischen religiösen Traditionen mit unterschiedlichem Verdichtungsgrad festgeschrieben werden. Mein Ziel ist ein rein heuristisches, nämlich Dynamiken zwischen verschiedenen Graden der Verdinglichung religiöser Traditionskomplexe analytisch besser fassbar zu machen.

\section{Zusammenfassung}

Bourdieu (1991) folgend wurden in diesem Artikel feldimmanente Aushandlungsprozesse bezüglich der Legitimität bestimmter Traditionsaspekte als konstitutiver Teil religiöser Traditionen aufgefasst. Solche Aushandlungsprozesse sind insbesondere an den Grenzen zwischen religiösen Traditionen besonders aktiv. Dabei sind diese Grenzen selbst auch Produkt der beschriebenen Aushandlungsprozesse und nicht fixiert. Anstatt, wie von Asad in AoI vorgenommen, vorschnell Referenzpunkte einer islamischen diskursiven Tradition zu bestimmen, sollten islamische Diskurse und Diskursbildungsprozesse vielmehr als prinzipiell offene

36 Hier stellt sich die Frage, ob moderne Grenzziehungen zwischen Religionen, wie sie zum Beispiel im (zunächst metasprachlichen) Weltreligionendiskurs vorgenommen wurden, als in einer semantischen Kontinuität mit diesem objektsprachlichen Modell der „Urdifferenzierung“ zwischen Religion/Christentum und Judentum verstanden werden können. Man könnte des Weiteren der Frage nachgehen, ob in diesem Zusammenhang auch der islamische Begriff din und die von ihm implizierten Unterscheidungen zwischen unterschiedlichen adyān, basierend auf einem idealtypischen islamischen dīn, schon eine Rolle spielen (vgl. Haußig 1999, 194).

37 Für ein instruktives Beispiel eines ethnographischen Ansatzes, der systematisch darum bemüht ist, Theoriebildung aus der objektsprachlichen Vorstellungswelt abzuleiten, siehe Cadena 2015. 
Aushandlungsprozesse innerhalb nicht beliebiger, aber dynamischer Machtgefüge betrachtet werden, die sich auf verschiedene traditionsbildende Autoritäten beziehen können.

Der vorgeschlagene Zugang zu religiösen Traditionen und Religionen verlagert den Blick von der Grenze zu der Kontaktzone, von Einflüssen zu Konversationen. Er stellt der dem Stammbaummodell innewohnenden Tendenz zur Hierarchisierung von Religionen ein dynamisches Verständnis der historischen Verhältnisse zwischen religiösen Traditionen unterschiedlichen Verdichtungsgrades entgegen. Die Frage nach den Ursprüngen des Alevitentums, nach der Trennlinie zwischen islamischem und nicht-islamischem Alevitentum, beziehungsweise zwischen islamischem und nicht-islamischem Sufismus erweist sich dann als Produkt eines Religionismus, dem es um die Fixierung von Religionsgrenzen und der essenzialistischen Festschreibung seiner Inhalte geht. Im Gegensatz hierzu sollte zum Beispiel das Problem der historischen Entwicklung des Alevitentums in einer Weise behandelt werden, die erkennbar macht, wie Ansprüche von Reinheit, Originalität und Authentizität als rhetorische Mittel zur Etablierung und Legitimation eines dominanten Diskurses eingesetzt werden. Darüber hinaus sollte es der hier vorgeschlagene Begriff von religiöser Tradition ermöglichen, zur Beschreibung und Analyse dynamischer Traditions- und Religionsbildungsprozesse jenseits der konstruktivistischen Kritik beizutragen.

Dass die Definitionsarbeit am Abstraktum Religion sowie an der Bestimmung einzelner Religionen nicht nur von wissenschaftlichem Interesse ist, sondern auch eine politische Dimension hat, wurde in den letzten Jahrzehnten nicht zuletzt von Vertreterinnen und Vertretern postkolonialer Ansätze in der Religionsforschung, die auf die Verschränkung wissenschaftlicher und politischer Interessen in der Essenzialisierung von Kulturen und Religionen hinweisen, immer wieder unterstrichen (siehe Brunotte 2009). Dieser Essenzialismus hat, wie zeitgenössische europäische Diskurse über „den Islam“ verdeutlichen, bisweilen auch eine rassistische Konnotation (Shooman und Spielhaus 2010; Schrode 2015). Die Reflexion über Prozesse der Verdichtung von religiösen Traditionen und über Grenzziehungsprozesse zwischen Religionen ist deshalb nicht nur von analytischem Interesse - nicht zuletzt, weil Diskurse über Grenzen zwischen Religionen im Zeitalter globaler „Flüchtlingskrisen“ vielerorts (un-)heilige Allianzen mit nationalistisch motivierten Grenzbefestigungsdiskursen eingehen. Die Religionswissenschaft sollte sich hier kritisch positionieren und sich auch der gesellschaftlichen Verantwortung ihrer Expertise zu Religion sowie Diskursen über Religion stellen.

Danksagung: Ich bedanke mich bei allen Teilnehmenden der beiden von Paula Schrode und ihrem Mitarbeiterstab organisierten Workshops in Bayreuth, in denen das Fundament für diese Aufsatzsammlung gelegt wurde, für Kommentare 
und Kritik an früheren Versionen dieses Beitrags. Der Aufsatz wurde mit Unterstützung durch die DFG-Kolleg-Forschergruppe Multiple Secularities: Beyond the West, Beyond Modernities fertiggestellt und profitierte auch von den dortigen Diskussionen. Ganz herzlichen Dank an Paula Schrode, Danijel Cubelic und Leyla Jagiella für die kritische und konstruktive Begleitung bei der Fertigstellung dieses Aufsatzes. Für weitere hilfreiche Hinweise bedanke ich mich bei Hubert Seiwert, Monika Wohlrab-Sahr, Judith Zimmermann und Foteine König sowie, für die letzte Überarbeitung des Texts, bei den Herausgebern der ZfR sowie bei den anonymen Gutachtern für ihre konstruktiven Anmerkungen und Verbesserungsvorschläge.

\section{Bibliographie}

Al Jerrahi, Sheikh N. 1994. „The Dream.” In American Jihad: Islam after Malcolm X, hg. v. Steven Barboza, 190-203. New York: Image Books Doubleday.

Anjum, Ovamir. 2007. „Islam as a Discursive Tradition: Talal Asad and His Interlocutors.“ Comparative Studies of South Asia, Africa and the Middle East 27:656-672.

Asad, Talal. 1986. The Idea of an Anthropology of Islam. Occasional papers series / Center for Contemporary Arab Studies, Georgetown University. Washington, D.C: Center for Contemporary Arab Studies Georgetown University.

Asad, Talal. 1993. Genealogies of Religion: Discipline and Reasons of Power in Christianity and Islam. Baltimore, Md. Johns Hopkins Univ. Press.

Asad, Talal. 2001. „Reading a Modern Classic: W. C. Smith's 'The Meaning and End of Religion'.“ History of Religions 40:205-222.

Asad, Talal. 2003. Formations of the Secular: Christianity, Islam, Modernity. Stanford, Calif. Stanford Univ. Press.

Asad, Talal. 2006. „Responses.“ In Powers of the Secular Modern: Talal Asad and His Interlocutors, hg. v. David Scott und Charles Hirschkind. 206-241. Stanford, Calif.: Stanford Univ. Press.

Becker, Adam H. 2015. Revival and Awakening: American Evangelical Missionaries in Iran and the Origins of Assyrian Nationalism. Chicago: University of Chicago Press.

Becker, Adam H. und Annette Y. Reed, Hg. 2003. The Ways that Never Parted: Jews and Christians in Late Antiquity and the Early Middle Ages. Tübingen: Mohr Siebeck.

Bisson, David. 2007. „Soufisme et tradition: L'influence de René Guénon sur l'islam soufi européen." Archives de Sciences Sociales des Religions 140:29-47.

Bourdieu, Pierre. 1991. „Genesis and Structure of the Religious Field.” Comparative Social Research 13:1-44.

Boyarin, Daniel. 1999. Dying for God: Martyrdom and the Making of Christianity and Judaism. Stanford, Calif: Stanford University Press.

Boyarin, Daniel. 2004. Border Lines: The Partition of Judaeo-Christianity. Philadelphia, Pa.: University of Pennsylvania Press. 
Brunotte, Ulrike. 2009. „Religion und Kolonialismus.“ In Europäische Religionsgeschichte: ein mehrfacher Pluralismus, hg. v. Hans Kippenberg, Jörg Rüpke und Kocku von Stuckrad, 339370. Göttingen: Vandenhoeck \& Ruprecht.

Cadena, Marisol de la. 2015. Earth Beings: Ecologies of Practice across Andean Worlds. Durham, NC: Duke Univ. Press.

Casanova, José. 1994. Public Religions in the Modern World. Chicago: University of Chicago Press.

Deleuze, Gilles und Félix Guattari. 1977. Rhizom. Berlin: Merve-Verlag.

Derrida, Jacques. 2002. „Faith and Knowledge: The Two Sources of 'Religion' at the Limits of Reason Alone.“ In Acts of Religion, hg. v. Gil Anidjar, 40-100. London: Routledge.

Dressler, Markus. 2008. „Religio-Secular Metamorphoses: The Re-Making of Turkish Alevism.“ Journal of the American Academy of Religion 76:280-311.

Dressler, Markus. 2009. „Pluralism and Authenticity: Sufi Paths in post-9/11 New York.“ In Sufis in Western Society: Global Networking and Locality, hg. v. Ron Geaves, Markus Dressler und Gritt Klinkhammer, 77-97. London: Routledge.

Dressler, Markus. 2010. „Between Legalist Exclusivism and Mysticist Universalism: Contested Sufi Muslim Identities in New York.“ Muslim World 100:431-451.

Dressler, Markus. 2013a. Writing Religion: The Making of Turkish Alevi Islam. New York: Oxford Univ. Press.

Dressler, Markus. 2013b. „Was ist das Alevitum? Die aktuelle Diskussion und historische Traditionslinien." In Ocak und Dedelik: Institutionen religiösen Spezialistentums bei den Aleviten, hg. v. Janina Karolewski, Robert Langer und Raoul Motika, 13-35. Frankfurt a. M.: Lang.

Dressler, Markus. 2018. „The Social Construction of Reality (1966) Revisited: Epistemology and Theorizing in the Study of Religion." Method and Theory in the Study of Religion, 16. March, Artikelvorabzugriff. Online: https://brill.com/view/journals/mtsr/aop/article-10.116315700682-12341434.xml

Dressler, Markus. „Modes of Religionization: A Constructivist Approach to Secularity.” Working Paper Series of the HCAS „Multiple Secularities - Beyond the West, Beyond Modernities” 7, Leipzig, February 2019. Online: https://www.multiple-secularities.de/media/wps7_dressler_religionization.pdf.

Ed. [1986]. „Madhhab”. In: Encyclopaedia of Islam, Second Edition, hg. v. P. Bearman, Th. Bianquis, C.E. Bosworth, E. van Donzel und W.P. Heinrichs. Online http://dx.doi.org/10.1163/1 573-3912_islam_SIM_8798.

Enver Behnan. 1927. „Kızılbaşlık. Esası Nedir?“ Büyük Gazete 26, 21. April, 12 f.

Gardet, L[ouis]. 2017. „Dīn.“ In Encyclopaedia of Islam. Second Edition, hg. v. P. Bearman, Th. Bianquis, C. E. Bosworth, E. van Donzel und W. P. Heinrichs. Online http://dx.doi.org/10.11 63/9789004206106_eifo_COM_0168.

Gölpınarlı, Abdülbâki. 1963. Alevî-ektasî Nefesleri. Istanbul: Remzi.

Grieve, Gregory P. und Richard Weiss. 2005. „Illuminating the Half-Life of Tradition: Legitimation, Agency, and Counter-Hegemonies.“ In Historicizing "Tradition“ in the Study of Religion, hg. v. Steven Engler und Gregory P. Grieve, 1-18. Berlin: De Gruyter.

Haddad, Yvonne Yazbeck. 1974. „The Conception of the Term Dīn In The Qur'ān.“ The Muslim World 64:114-123.

Haußig, Hans-Michael. 1999. Der Religionsbegriff in den Religionen: Studien zum Selbst- und Religionsverständnis in Hinduismus, Buddhismus, Judentum und Islam. Berlin: Philo. 
Hermansen, Marcia. 2000. „Hybrid Identity Formations in Muslim America: The Case of American Sufi Movements.“ The Muslim World 90:158-197.

Houston, Christopher. 2009. „The Islam of Anthropology.“ The Australian Journal of Anthropology 20:198-212.

Hoyland, Robert. 2012. „Early Islam as a Late Antique Religion.“ In The Oxford Handbook of Late Antiquity, hg. v. Scott Fitzgerald Johnson, 1053-1077. New York: Oxford Univ. Press.

Hoyland, Robert. 2015. The Late Antique World of Early Islam: Muslims among Christians and Jews in the East Mediterranean. Princeton, NJ: The Darwin Press.

Hughes, Aaron W. 2017. Shared Identities: Medieval and Modern Imaginings of Judeo-Islam. New York: Oxford Univ. Press.

Iqbal, Basit K. 2017. „Thinking about Method: A Conversation with Talal Asad.“ Qui Parle: Critical Humanities and Social Sciences 26:195-218.

Karamustafa, Ahmet T. 2017. „Islamic Dīn as an Alternative to Western Models of 'Religion'.” In Religion, Theory, Critique: Classic and Contemporary Approaches and Methodologies, hg. v. Richard King, 163-171. New York: Columbia Univ. Press.

Krech, Volkhard. 2012. „Dynamics in the History of Religions: Preliminary Considerations on Aspects of a Research Programme." In Dynamics in the History of Religions between Asia and Europe: Encounters, Notions, and Comparative Perspectives, hg. v. Volkhard Krech und Marion Steinicke, 15-70. Leiden: Brill.

Luhmann, Niklas. 2013. A Systems Theory of Religion. Translated by David A Brenner with Adrian Hermann. Stanford: Stanford Univ. Press.

Masuzawa, Tomoko. 2005. The Invention of World Religions: Or, how European Universalism Was Preserved in the Language of Pluralism. Chicago: Univ. of Chicago Press.

Neuwirth, Angelika. 2010. Der Koran als Text der Spätantike: Ein europäischer Zugang. Berlin: Verl. der Weltreligionen.

Öngören, Reşat. 2011. „Tarikat.“ In Türkiye Diyanet Vakfı ìlâm Ansiklopedisi. Bd. 40, 95-105. Istanbul: Türkiye Diyanet Vakfı İslâm Araştırmaları Merkezi.

Riesebrodt, Martin. 2004. „Überlegungen zur Legitimität eines universalen Religionsbegriffs.” In Religion im kulturellen Diskurs: FS für Hans G. Kippenberg zu seinem 65. Geburtstag, hg. v. Brigitte Luchesi und Kocku von Stuckrad, 127-149. Berlin: de Gruyter.

Riesebrodt, Martin. 2007. Cultus und Heilsversprechen: Eine Theorie der Religionen. München: Beck.

Saar, Martin. 2008. „Understanding Genealogy: History, Power, and the Self.“ Journal of the Philosophy of History 2:295-314.

Salahi, Mehmed. 1895-1904. Kamus-i Osmani. Istanbul: Mahmud Bey Matbaası.

Sami, Şemseddin. 2017. Kâmûs-ı Türkî. Öğrenciye Özel Tıpkıbasım. Istanbul: Çağrı Yay.

Schielke, Samuli. 2010. Second Thoughts about the Anthropology of Islam, or how to Make Sense of Grand Schemes in Everyday Life. ZMO working paper 2.2010. Berlin: Zentrum Moderner Orient.

Schrode, Paula. 2010. Sunnitisch-islamische Diskurse zu Halal-Ernährung: Konstituierung religiöser Praxis und sozialer Positionierung unter Muslimen in Deutschland. Würzburg: ErgonVerl.

Schrode, Paula. 2015. „Islam und Rassismus in Deutschland.“ In Neue alte Rassismen?: Differenz und Exklusion in Europa nach 1989, hg. v. Gesine Drews- Sylla und Renata Makarska, 45-68. Bielefeld: Transcript-Verl.

Schulze, Reinhard. 2015. Der Koran und die Genealogie des Islam. Basel: Schwabe Verl. 
Scott, David. 2006. „The Tragic Sensibility of Talal Asad.“ In Powers of the Secular Modern: Talal Asad and His Interlocutors, hg. v. David Scott und Charles Hirschkind, 134-153. Stanford, Calif.: Stanford Univ. Press.

Scott, David und Charles Hirschkind. 2006. „Introduction: The Anthropological Skepticism of Talal Asad.“ In Powers of the Secular Modern: Talal Asad and His Interlocutors, hg. v. David Scott und Charles Hirschkind, 1-11. Stanford, Calif.: Stanford Univ. Press.

Shooman, Yasemin und Riem Spielhaus. 2010. „The Concept of the Muslim Enemy in the Public Discourse." In Muslims in the West after 9/11: Religion, Politics, and Law, hg. v. Jocelyne Cesari, 198-228. London: Routledge.

Smith, Wilfred Cantwell. 1991. The Meaning and End of Religion. Minneapolis: Fortress Press.

Tümer, Günay. 1994. „Din.“ In Türkiye Diyanet Vakfı İslâm Ansiklopedisi. Bd. 9, 312-320. Istanbul: Türkiye Diyanet Vakfı İslâm Araştırmaları Merkezi.

Tweed, Thomas A. 2006. Crossing and Dwelling: A Theory of Religion. Cambridge: Harvard Univ. Press.

Üzüm, illyas. 2004. „Mezhep.“ In Türkiye Diyanet Vakfı İslâm Ansiklopedisi. Bd. 29, 526-534. Istanbul: Türkiye Diyanet Vakfı İslâm Araştırmaları Merkezi.

Murre-van den Berg, Heleen, Jan J. Ginkel und Theo M. van Lint, Hg. 2005. Redefining Christian Identity: Cultural Interaction in the Middle East since the Rise of Islam. Leuven: Peeters.

Wick, Peter. 2012. „Dynamic Approaches to Antique Religions in the East and West: Beyond Centres and Boundaries." In Dynamics in the History of Religions between Asia and Europe: Encounters, Notions, and Comparative Perspectives, hg. v. Volkhard Krech und Marion Steinicke, 73-83. Leiden: Brill.

Wilson, M. Brett. 2007. „The Failure of Nomenclatura: The Concept of 'Orthodoxy' in the Study of Islam.“ Comparative Islamic Studies 3:169-194.

Wunn, Ina. 2018. Barbaren, Geister, Gotteskrieger. Die Evolution der Religionen - entschlüsselt. Springer: Berlin. 